\title{
Wave optical algorithm for creating digitally synthetic holograms of three-dimensional surface objects
}

\author{
K. Matsushima and A. Kondoh \\ Department of Electrical Engineering, Kansai University \\ Yamate-cho 3-3-35, Suita, Osaka 564-8680, Japan
}

\begin{abstract}
Progress in a novel wave-optical algorithm for synthesizing object waves of three-dimensional surface objects is reported. The algorithm has been proposed last year and has a feature of wave-optical calculation in diffraction by object surfaces. Numerical simulation of diffraction is implemented by a method based on coordinates rotation in Fourier spectrum. Source fields on a surface of objects are given as complex property functions defined on each object surface. These property functions provide information on each surface, such as the shape and diffusiveness. General formulation of the algorithm has been presented, but its numerical implementation was limited only to the cases of single axis rotation of plane surfaces.

In this investigation the limitation is removed, i.e. two-axes rotation of plane surfaces is presented and reconstruction of holograms of 3-D objects composed of rotated planes are demonstrated. Furthermore, compensation of surface brightness is discussed for accurate shading objects.
\end{abstract}

Keywords: computer-generated hologram, digitally synthetic hologram, surface model, shading

\section{INTRODUCTION}

In digitally synthetic holograms (or computer-generated holograms; CGH), some object models and methods for synthesizing object waves emitted from the three-dimensional (3-D) objects has been proposed to create real auto-stereoscopic 3-D images. One of the most important models is the layer model, in which the object is sliced into a plane parallel to the hologram and light fields emitted from every plane are wave-optically calculated as ordinary diffraction such as Fresnel diffraction. While the method has a definite advantage of relatively short computation time owing to utilization of FFT especially in full-parallax hologram, there is a major drawback to realistic rendering of 3-D objects such as shading, shadowing, texture-mapping and so on.

An alternative model recently used is the point source model, which is sometimes referred to as the raytracing method in CGH. ${ }^{1,2}$ A 3-D object is considered as a collection of numerous point sources of light in the model, and the object wave is obtained by superimposing all spherical waves from point sources in the hologram plane. Since computation time of the ray-tracing is proportional to both numbers of point sources and sampling points in the hologram, slow computation is a major disadvantage of the method, especially in full-parallax holograms. To overcome the problem of computation time in the ray-tracing some algorithm has been proposed, such as real-valued fringe intensity, ${ }^{2,3}$ utilization of geometric symmetry ${ }^{4}$ and special hardware, ${ }^{5-7}$ lookup table ${ }^{3}$ recurrence formulas ${ }^{8}$ and difference formulas. ${ }^{9}$

One another method we refer it to as the wave optical algorithm in surface model has been suggested in the last meeting. ${ }^{10}$ In the method an object is composed of planar surfaces and the object wave is waveoptically calculated as reflection and diffraction by the tilted surfaces. The mathematical and physical basics have been presented, but a hologram of planar objects rotated only upon a single axis has been discussed and its reconstruction was demonstrated. This report follows with creating holograms for true 3-D objects made up from planes rotated on two-axes, as well as shading the objects by using a numerical model of brightness of reconstructed planar surfaces.

Further author information: (Send correspondence to Matsushima)

Matsushima: E-mail: matsu@kansai-u.ac.jp, Telephone: +81-6-6368-0933 


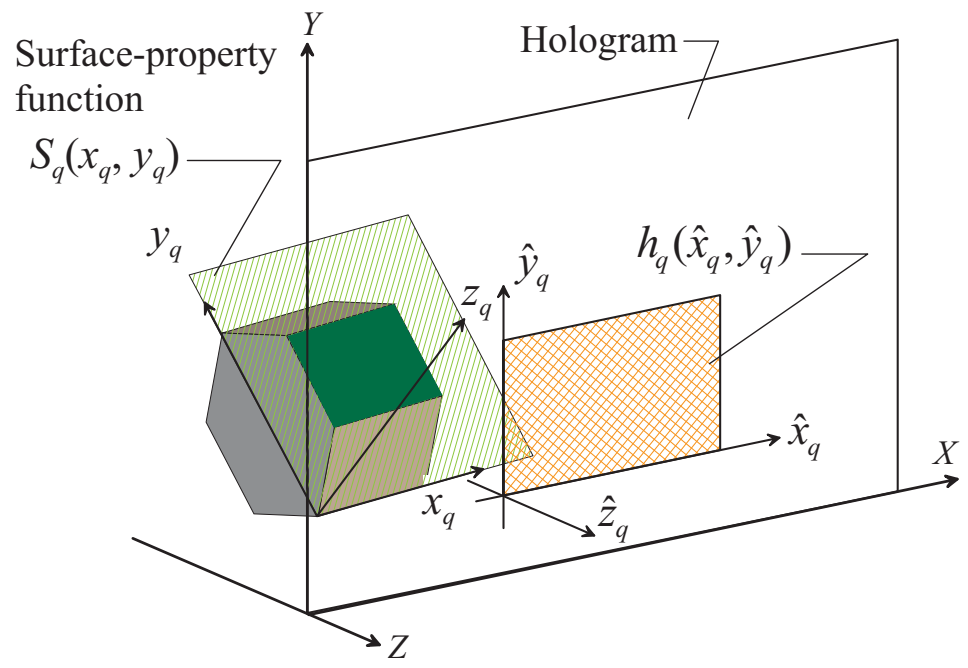

Figure 1. An object model and coordinate system used to synthesize object waves.

\section{OBJECT MODEL AND SURFACE PROPERTY FUNCTION}

Coordinates system and geometry used in this investigation is shown in Fig. 1. The global coordinates, referred to as hologram coordinates, is denoted by $(X, Y, Z)$, and the hologram is placed on the $(X, Y, 0)$ plane. Local coordinates defined for each surface of the object are $\left(x_{q}, y_{q}, z_{q}\right)$ in a surface $q$. All local coordinates are defined so that the planar surface is placed on the $\left(x_{q}, y_{q}, 0\right)$ plane. Furthermore, we define a complex function $s_{q}\left(x_{q}, y_{q}\right)$ in the $\left(x_{q}, y_{q}, 0\right)$ plane to give the surface $q$ its properties such as the shape, brightness, diffusiveness, feel of material, textures and so on. These complex functions are referred to as the surface property function. In addition, we define one another coordinates, the parallel local coordinates $\left(\hat{x}_{q}, \hat{y}_{q}, \hat{z}_{q}\right)$, in which the origin is identical to the local coordinates but all axes are parallel to that of the hologram coordinates.

All surface property functions are defined in the following form:

$$
s_{q}\left(x_{q}, y_{q}\right)=a_{q}\left(x_{q}, y_{q}\right) p_{q}\left(x_{q}, y_{q}\right) \Psi\left(x_{q}, y_{q}\right)
$$

where $a_{q}\left(x_{q}, y_{q}\right)$ is a real function and mainly keeps the shape of the surface $q$ and its texture. A surface property function defined only by the factor $a_{q}\left(x_{q}, y_{q}\right)$ do not have any diffusiveness, just like as a slide projector, and therefore it must be multiplied by a given diffusive phase function; a diffuser given as the complex function:

$$
\Psi\left(x_{q}, y_{q}\right)=\exp \left[i k \phi_{d}\left(x_{q}, y_{q}\right)\right]
$$

Here, we used a digital diffuser proposed for Fourier holograms ${ }^{11}$ to the phase function $\phi_{d}\left(x_{q}, y_{q}\right)$.

If a surface property function are given as $a_{q}\left(x_{q}, y_{q}\right) \Psi\left(x_{q}, y_{q}\right)$, the carrier frequency of the object wave are placed at the zero frequency in the local coordinates. This means that the light wave of the surface is emitted in the direction perpendicular to the surface. Therefore, a phase of the plane wave propagating along the $Z$ axis (i.e. in the direction perpendicular to the hologram) should be multiplied by the two factors given above. The plane wave is written by

$$
p_{q}\left(x_{q}, y_{q}\right)=\exp \left[i k\left(\cos \alpha_{q} x_{q}+\sin \beta_{q} y_{q}\right)\right]
$$

where $\cos \alpha_{q}$ and $\cos \beta_{q}$ are the direction cosines in $X$ and $Y$ axis, respectively.

The surface property function given above is transformed into the complex amplitude $h_{q}\left(\hat{x}_{q}, \hat{y}_{q}\right)$ in the local parallel coordinates by the method described in Ref. 10,12. This transformation written as

$$
h_{q}\left(\hat{x}_{q}, \hat{y}_{q}\right)=\mathcal{R}_{\theta_{x} \theta_{y} \theta_{z}}\left\{s_{q}\left(x_{q}, y_{q}\right)\right\}
$$



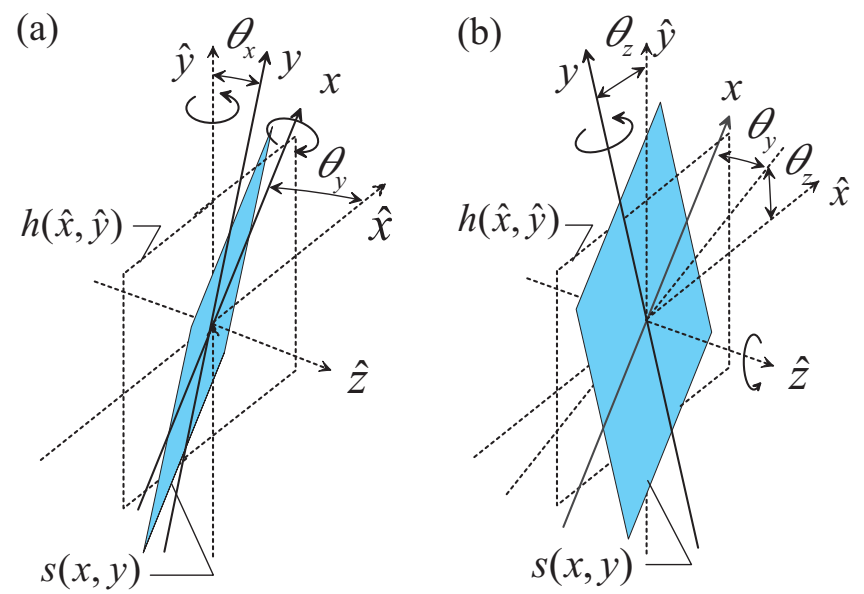

Figure 2. Two types of rotation upon two-axes; rotating $(\hat{x}, \hat{y}, \hat{z})$ coordinates upon the $\hat{y}$-axis prior to the $x$-axis (a), rotating upon the $\hat{z}$-axis prior to the $y$-axis (b)
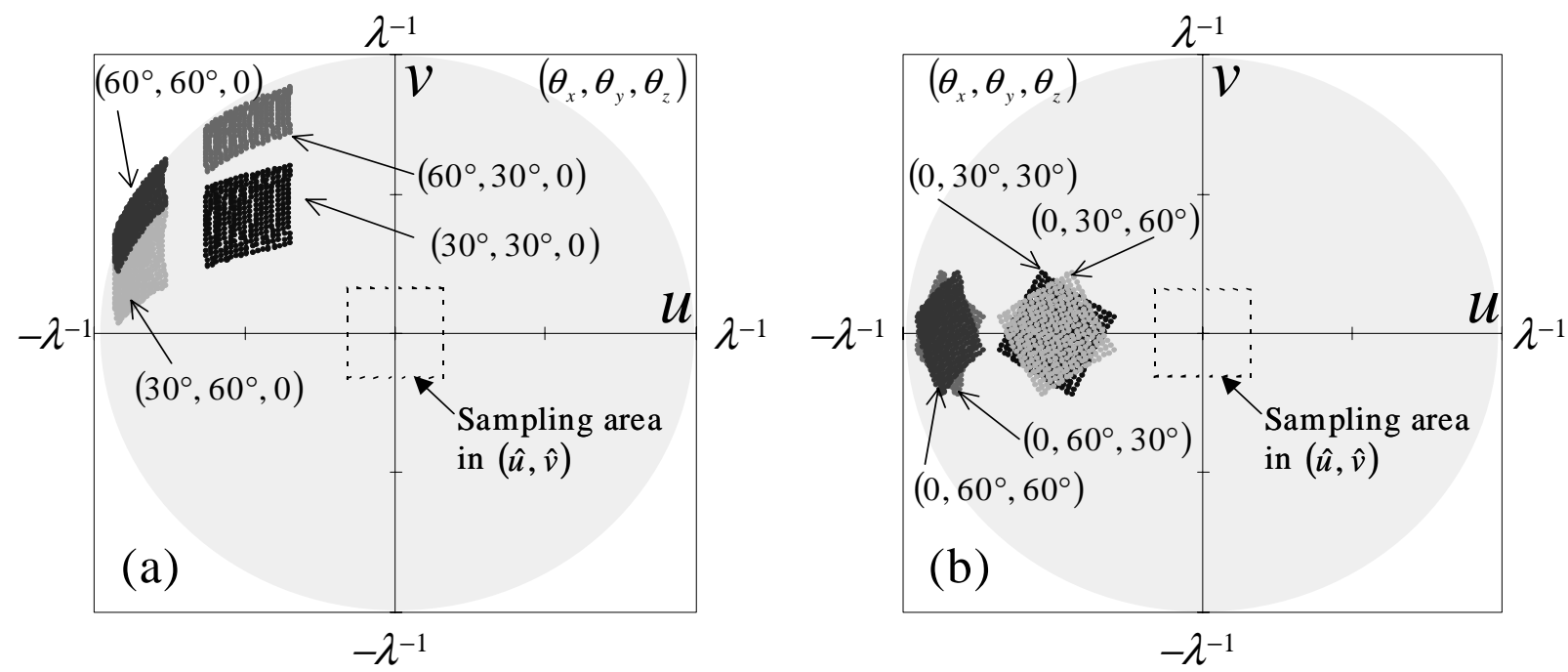

Figure 3. Distribution of sampling points in $(u, v)$. The rotation scheme in (a) and (b) agrees with that of Fig. 2.

where $\theta_{x}, \theta_{y}$ and $\theta_{z}$ are rotation angles upon each axis. Finally, fields emitted from every plane are superimposed in the hologram plane as follows:

$$
H(X, Y)=\sum_{q} h_{q}\left(\hat{x}_{q}, \hat{y}_{q}\right)
$$

\section{COORDINATES ROTATION ON TWO-AXES}

There are several degrees of freedom to define local coordinates for each surface. Fig. 2, for example, shows two types of rotation on two-axes. In Fig. 2(a) the parallel local coordinates $(\hat{x}, \hat{y}, \hat{z})$ is transformed into the local coordinates by rotating coordinates upon $\hat{y}$ axis prior to $x$ axis, while the coordinates is rotated upon $\hat{z}$ axis prior to $y$ axis in (b). When a position vector $\mathbf{r}$ in the local coordinates is transformed into $\hat{\mathbf{r}}$ in the parallel local coordinates by transformation matrix $T$, the transformation is written as

$$
\hat{\mathbf{r}}=\operatorname{Tr} .
$$


The inverse transformation matrix in the case indicated in Fig. 2(a) is given by

$$
\begin{aligned}
\mathbf{T}^{-1} & =\mathbf{R}_{x}\left(\theta_{x}\right) \mathbf{R}_{y}\left(\theta_{y}\right) \\
& =\left[\begin{array}{ccc}
\cos \theta_{y} & 0 & -\sin \theta_{y} \\
\cos \theta_{x} \cos \theta_{y} & \sin \theta_{x} & -\cos \theta_{x} \sin \theta_{y} \\
-\sin \theta_{x} \cos \theta_{y} & \cos \theta_{x} & \cos \theta_{x} \sin \theta_{y}
\end{array}\right] .
\end{aligned}
$$

The inverse matrix for Fig. 2(b) is also given by

$$
\begin{aligned}
\mathbf{T}^{-1} & =\mathbf{R}_{y}\left(\theta_{y}\right) \mathbf{R}_{z}\left(\theta_{z}\right) \\
& =\left[\begin{array}{ccc}
\cos \theta_{y} \cos \theta_{z} & \cos \theta_{y} \sin \theta_{z} & -\sin \theta_{y} \\
-\sin \theta_{z} & \cos \theta_{z} & 0 \\
\sin \theta_{y} \cos \theta_{z} & \sin \theta_{y} \sin \theta_{z} & \cos \theta_{y}
\end{array}\right]
\end{aligned}
$$

Selection of these rotation schemes are not significant in the aspect of the mathematical transformation of coordinates, nevertheless one should carefully choose the rotation scheme, because an interpolation of Fourier spectrum is necessary for numerical calculation in the coordinates rotation. Since an object wave is typically sampled on an equidistant grid in the hologram plane, sampling points of the spectrum in $(\hat{u}, \hat{v})$ coordinates is also distributed on an equidistant grid within a square area, as shown in Fig. 3. The sampling points after any rotational transformation, however, are not positioned at even intervals, and its envelope does not shape square any longer. Fig. 3(a) and (b) shows distribution of sampling points in the local coordinates after rotating coordinates by using the way indicated in Fig. 2(a) and (b), respectively.

The rotation scheme in Fig. 2(b), described by matrix (10), is adopted in this report, because it seems that distortion of sampling distribution in Fig. 3(b) is rather less than that in (a). Jacobian necessary for rotational transformation by the matrix (10) (See Appendix A of Ref. 10) is given by

$$
J(\hat{u}, \hat{v})=\sin \theta_{y} \cos \theta_{z} \frac{\hat{u}}{\hat{w}(\hat{u}, \hat{v})}+\sin \theta_{y} \sin \theta_{z} \frac{\hat{v}}{\hat{w}(\hat{u}, \hat{v})}+\cos \theta_{y} .
$$

\section{OBJECT WAVES FROM THREE-DIMENSIONAL OBJECTS}

Object waves emitted from 3-D objects can be synthesized by superimposing object waves emitted by planes constructing the object. A cube, for example, consists of three square planes*, as shown in Fig 4(a). An optical

${ }^{*}$ In this report we do not treat methods for removing hidden surfaces, and thus, such hidden surfaces are originally not taken into account.

(a)

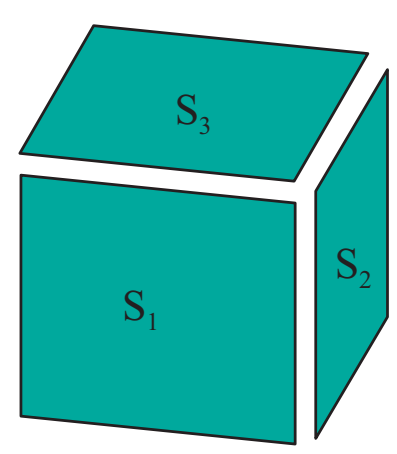

(b)

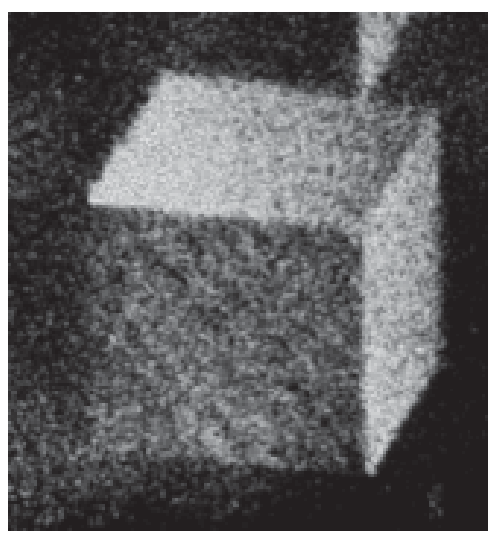

Figure 4. A cube built by plane surfaces (a) and an optical reconstruction of a fabricated hologram (b). The dimension of the cube is $6 \times 6 \times 6 \mathrm{~mm}^{3}$. 
reconstruction of a fabricated hologram of the cube is shown in Fig 4(b). A cube is optically reconstructed as expected, however the cube is shaded unexpectedly. All amplitudes of the surface property functions were a same constant, i.e. $a_{q}\left(x_{q}, y_{q}\right) \equiv a_{0}$, nevertheless the reconstructed cube has a definite shade. This shading is unexpected one and should be compensated in order to shade object accurately.

\subsection{Numerical model for brightness of reconstructed surfaces}

For compensation of the unexpected shading it is necessary to investigate which and how parameters of an object surface relate to brightness of the surface on reconstruction. Figure 5 is a theoretical model to predict brightness of a surface, represented by the sampled property function.

Suppose that the amplitude of a surface property function is a constant, i.e. $a(x, y) \equiv a_{0}$ and $|s(x, y)|^{2}=a_{0}^{2}$ gives optical intensity on the surface. In that cases radiant flux $\Phi$ of a small area $\delta A$ on the surface is given by

$$
\begin{aligned}
\Phi & =\iint_{\delta A}|s(x, y)|^{2} d x d y \\
& =\delta A \sigma a_{0}^{2},
\end{aligned}
$$

where $\sigma$ is the surface density of sampling. We assumed that almost the whole light emitted from the small area $\delta A$ is bound in a solid angle given by

$$
\begin{aligned}
\Omega & =\frac{A}{r^{2}}=\frac{\pi\left(r \tan \varphi_{d}\right)^{2}}{r^{2}} \\
& =\pi \tan ^{2} \varphi_{d},
\end{aligned}
$$

where $\varphi_{d}$ is a parameter representing degree of the diffusiveness of the diffuser function $\Psi\left(x_{q}, y_{q}\right)$. Furthermore, assuming that light is diffused almost uniformly i.e. $d \Phi / d A \simeq \Phi / A$, brightness of the surface, observed at angle of $\theta$ with the normal vector, is given by

$$
\begin{aligned}
L & =\frac{d \Phi / d \Omega}{\cos \theta \delta A} \\
& =\frac{\sigma a_{0}^{2}}{\pi \tan ^{2} \varphi_{d} \cos \theta} .
\end{aligned}
$$

As a result, brightness of the surface depends on the surface density of sampling, diffusiveness of the diffuser function, and amplitude of the surface property function. In addition, brightness varies dependently on the observation angle. This means that brightness of the surface changes according to the direction of the surface and causes unexpected shade.

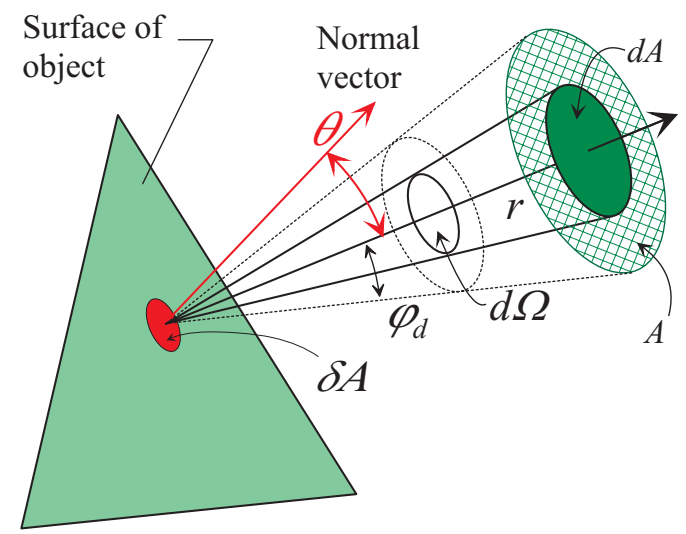

Figure 5. A theoretical model for predicting brightness of a planar surface represented by the surface property function. 
A simple theoretical model is discussed so far, therefore Eq. (15) is only partially fit for brightness of optically reconstructed surfaces of real holograms. Since brightness given in Eq. (15) diverges in limit $\theta \rightarrow \pi / 2$ and any actual hologram cannot produce infinite brightness for its reconstructed surface, Eq. (15) is not sufficient for the model used for compensation. To avoid the divergence of brightness in Eq. (15) we a priori introduced an angle factor $(1+\gamma) /(\cos \theta+\gamma)$ instead of $1 / \cos \theta$, i.e. supposed brightness is given as

$$
L=\frac{\sigma a_{0}^{2}}{\pi \tan ^{2} \varphi_{d}} \frac{(1+\gamma)}{(\cos \theta+\gamma)},
$$

where $\gamma$ is a parameter that plays a role to avoid the divergence of brightness and prevent overcompensation as a result. The $\gamma$ is dependent on actual methods to fabricate holograms such as encoding of object waves or property of recording materials and thus should be determined experimentally. Note that the angle factor in Eq. (16) is unity in $\theta=0$ and $1+1 / \gamma$ in $\theta=\pi / 2$.

\subsection{Compensation of brightness and shading object}

Shading object is based on simple Lambert's low and the diffused reflection model. Brightness of a surface $q$, of which the normal vector is at an angle of $\hat{\theta}$ with the vector of illumination, is given by

$$
L_{q}=L_{0}\left(\cos \hat{\theta}_{q}+l_{e}\right),
$$

where $l_{e}$ is the ratio of surrounding light to illuminated one and $L_{0}$ is brightness in $\hat{\theta}_{q}=0$ and $l_{e}=0$. By substituting $L_{q}$ in Eq. (17) into $L$ in Eq. (16), amplitude $a_{q}$ of a surface $q$ is given as follows:

$$
\begin{aligned}
& a_{q}=A_{0} \sqrt{\frac{\left(\cos \hat{\theta}_{q}+l_{e}\right)\left(\cos \theta_{q}+\gamma\right)}{1+\gamma}}, \\
& A_{0} \equiv \sqrt{\frac{L_{0} \pi \tan ^{2} \varphi_{d}}{\sigma}} .
\end{aligned}
$$

Here, the observation angle $\theta$ is replaced by an angle $\theta_{q}$ formed between the surface $q$ and the hologram.

\section{OPTICAL RECONSTRUCTION OF FABRICATED HOLOGRAMS OF THREE-DIMENSIONAL OBJECTS}

Digitally synthetic holograms were fabricated by a special purpose printer for drawing holograms. Parameters used to synthesize object waves and fabricate holograms are summarized in Table 1.

Table 1. Parameters used to synthesize object waves and fabricate holograms.

\begin{tabular}{ll}
\hline \hline Object and its surface property function & \\
\hline Sampling pitch & $2 \mu \mathrm{m} \times 2 \mu \mathrm{m}$ \\
Dimensions & $6 \mathrm{~mm} \times 6 \mathrm{~mm} \times 6 \mathrm{~mm}$ (cube) \\
& $8 \mathrm{~mm}$ in diameter (hexagonal pyramid) \\
Distance from hologram & $100 \mathrm{~mm}$ \\
\hline \hline Hologram & $8192 \times 4096$ \\
\hline Number of pixels & $2 \mu \mathrm{m} \times 4 \mu \mathrm{m}$ \\
Pixel size & $632.8 \mathrm{~nm}$ \\
Reconstruction wavelength & Point oriented multi-level amplitude \\
Coding & Plane wave with incidence angle of $4^{\circ}$ \\
Reference wave &
\end{tabular}



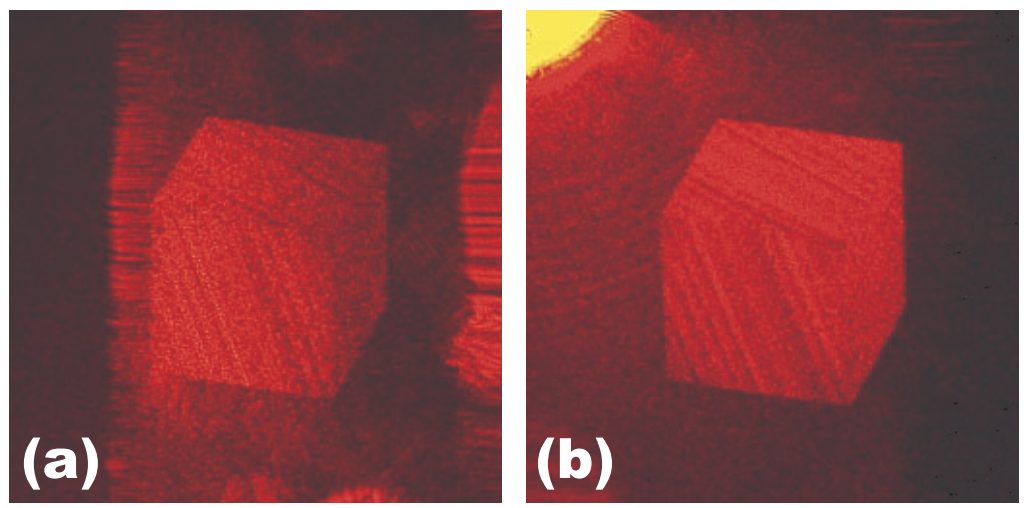

Figure 6. Optical reconstructions of cubic objects without illumination. Brightness of each surface is compensated in $\gamma=0(\mathrm{a})$ and $0.5(\mathrm{~b})$
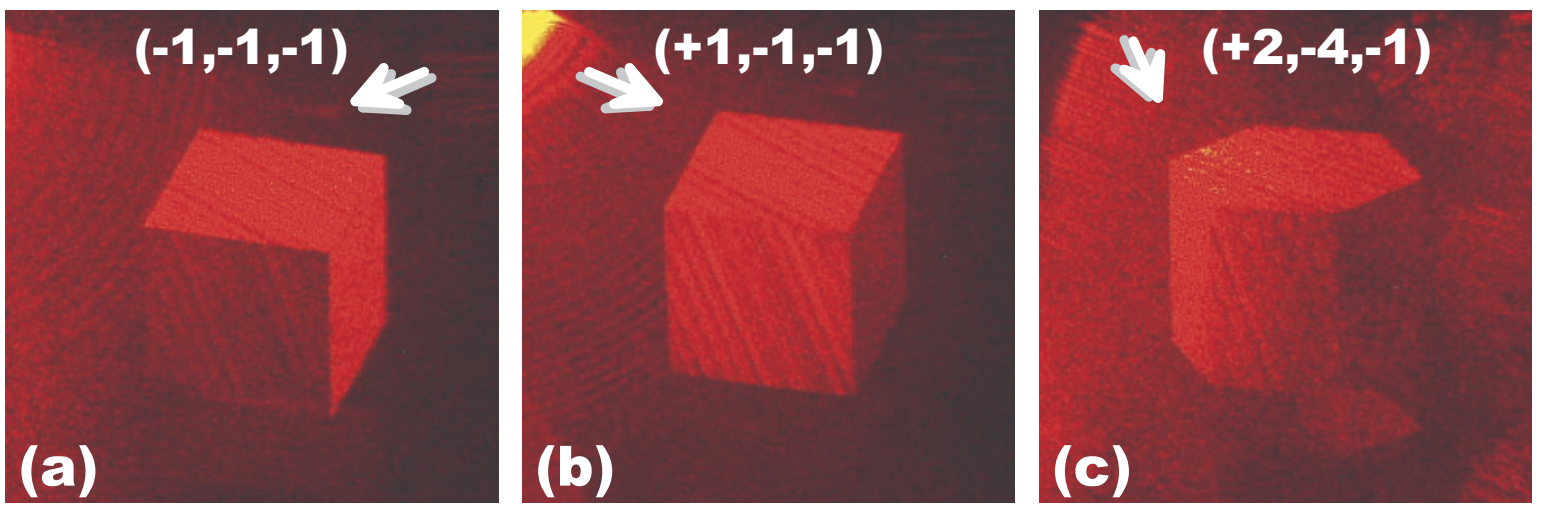

Figure 7. Optical reconstructions of 3-D objects shaded with illumination light; cubes illuminated from the upper right in $l_{e}=0$ (a) and the upper left in $l_{e}=0.7(\mathrm{~b})$, and a hexagonal prism $l_{e}=0.5$ (c). Brightness of every object is compensated in $\gamma=0.5$. An arrow and figures in parenthesis indicates the illumination vector.

Firstly several holograms of cubic objects without illumination light was fabricated to determine the value of the parameter $\gamma$. Figure 6 (a) shows optical reconstruction of a hologram with $\gamma=0$. In contrast to Fig. 4(b), the surface placed at a large angle with the hologram is dark because of overcompensation caused by $\gamma=0$. Figure 6(b) is also of a cubic object without illumination, but of which brightness is compensated in $\gamma=0.5$. Differences of brightness disappear because of proper compensation of brightness, and borders between surfaces are no longer found out.

Figures 7(a)-(c) shows optical reconstruction of 3-D objects shaded by illumination light, of which brightness is all compensated in $\gamma=0.5$. As was expected by arrows indicating illumination vectors in the figures, object surfaces are shaded in the reconstruction.

\section{CONCLUSION}

A detailed method for two-axes rotation of plane surfaces has been presented. Object waves emitted from three-dimensional objects composed of several rotated planes were digitally synthesized and reconstructions of the fabricated hologram were demonstrated. Furthermore, the surface brightness dependent on the observation angle as well as its compensation was discussed. 


\section{ACKNOWLEDGMENTS}

Authors acknowledge S. Yamanaka and H. Miyauchi for their assistance in fabricating holograms.

\section{REFERENCES}

1. J. P. Waters, "Holographic image synthesis utilizing theoretical methods," Appl. Phys. Lett. 9, pp. 405-407, 1966.

2. A. D. Stein, Z. Wang, and J. J. S. Leigh, "Computer-generated holograms: A simplified ray-tracing approach," Computers in Physics 6, pp. 389-392, 1992.

3. M. Lucente, "Interactive computation of holograms using a look-up table," J. Electronic Imaging 2, pp. 2834, 1993.

4. J. L. Juárez-Pérez, A. Olivares-Pérez, and R. Berriel-Valdos, "Nonredundant calculation for creating digital Fresnel holograms," Appl. Opt. 36, pp. 7437-7443, 1997.

5. A. Ritter, J. Böttger, O. Deussen, M. König, and T. Strothotte, "Hardware-based rendering of full-parallax synthetic holograms," Appl. Opt. 38, pp. 1364-1369, 1999.

6. T. Ito, T. Yabe, M. Okazaki, and M. Yanagi, "Special purpose computer holography HORN-1 for reconstruction of virtual image in three dimensions," Comput. Phys. Commun. 82, pp. 104-110, 1994.

7. T. Ito, H. Eldeib, K. Yoshida, S. Takahashi, T. Yabe, and T. Kunugi, "Special purpose computer for holography HORN-2," Comput. Phys. Commun. 93, pp. 13-20, 1996.

8. K. Matsushima and M. Takai, "Recurrence formulas for fast creation of synthetic three-dimensional holograms," Appl. Opt. 39, pp. 6587-6594, 2000.

9. H. Yoshikawa, S. Iwase, and T. Oneda, "Fast computation of Fresnel holograms employing difference," SPIE Proc. Practical Holography XIV and Holographic Materials VI \#3956, pp. 48-55, 2000.

10. K. Matsushima, H. Schimmel, and F. Wyrowski, "New creation algorithm for digitally synthesized holograms in surface model by diffraction from tilted planes," SPIE Proc. Practical Holography XVI and Holographic Materials VIII \#4659, pp. 53-60, 2002.

11. R. Bräuer, F. Wyrowski, and O. Bryngdahl, "Diffusers in digital holography," J. Opt. Soc. Am. A8, p. 572, 1991.

12. K. Matsushima, H. Schmmel, and F. Wyrowski, "Fast calculation method for optical diffraction on tilted planes using the angular spectrum of plane waves," (to be published). 